\title{
Modifications des représentations sociales de l'autisme et introduction du concept « autism-friendly »
}

\section{Brigitte Chamak}

La manière de concevoir le handicap a subi d'importants changements dans les années 2000. La distinction entre maladie et handicap s'est estompée avec la reconnaissance des conséquences handicapantes des maladies chroniques, des processus de vieillissement, des pathologies psychiatriques («handicap psychique ») et l'importance accrue accordée aux répercussions sociales des maladies. La classification internationale du fonctionnement, du handicap et de la santé ( $\mathrm{CIH}-2)$ de l'OMS (2001) définit actuellement le handicap comme une restriction de la participation sociale des personnes résultant de l'interaction entre des caractéristiques individuelles et des facteurs environnementaux. Cet élargissement de la notion de handicap rejoint l'élargissement des critères diagnostiques de nombreuses pathologies classées dans les maladies mentales, comme l'autisme, l'hyperactivité, le syndrome bipolaire ou la phobie sociale. Avec l'exemple de l'autisme, je tenterai de montrer comment la volonté de déstigmatiser l'autisme a abouti à une focalisation sur ceux qui présentent les formes les moins sévères et comment ces personnes se sont mobilisées pour redéfinir leur condition comme une différence.

Bien que l'autisme soit source d'incapacités, de limitations d'activité et de restriction de la participation sociale, sa reconnaissance officielle en tant que source de handicap, en France, est récente (loi Chossy, 1996). Forte revendication des associations de parents, rebaptiser handicap ce qui était qualifié de maladie psychiatrique, était perçu comme un moyen de déstigmatiser l'autisme. Cependant, l'attention et la médiatisation accrue orientées vers les personnes présentant un syndrome d'Asperger, signent le passage d'un extrême à l'autre. Alors que les représentations de l'autisme étaient très négatives avec une description des seules déficiences et incapacités, aujourd'hui l'accent est mis sur les capacités singulières, la mémoire étonnante ou les aptitudes de calcul exceptionnelles de certains d'entre eux.

Autrefois considéré comme une maladie rare, sévère et incurable, l'autisme est devenu un syndrome aux contours flous incluant des sujets sans langage avec déficience intellectuelle et des personnes avec langage aux capacités cognitives importantes mais présentant de réelles difficultés dans les interactions sociales. Les témoignages de certains d'entre eux ont été très diffusés. Les personnages avec syndrome d'Asperger se sont multipliés dans les films et les séries télévisées (Chamak, 2013). Leurs difficultés à comprendre les codes sociaux et les situations d'incompréhension qui en découlent ont été mises en scène. Le stéréotype de l'autiste "geek », as de l'informatique, s'est rapidement répandu. L'introduction du syndrome d'Asperger dans la catégorie "autisme " a modifié les images associées à l'autisme. Des adultes qui se sentaient différents mais ne comprenaient pas en quoi consistait leur différence se sont reconnues dans la description du fonctionnement autistique. Ils ont témoigné de leur expérience et ont tenté de changer l'image négative associée à l'autisme. Un nouveau mouvement social a émergé célébrant la " neurodiversité » en redéfinissant l'autisme comme une différence et non comme une maladie ou un handicap (Chamak, 2008, 2010 ; Ortega, 2009).

Mon travail de recherche à long terme a consisté à étudier les changements de définition de l'autisme liés aux modifications des classifications des maladies mentales, et à en analyser les conséquences. L'introduction de personnes aux capacités langagières et cognitives dans la catégorie a permis à ces personnes d'exprimer leur point de vue. Ainsi, la parole qui était jusque-là limitée à celle des professionnels et des parents s'est élargie. L'analyse des sites

Brigitte Chamak, sociologue et historienne des sciences ; Centre de Recherche Médecine, Sciences, Santé, Société (CERMES3) ; INSERM U988 - CNRS UMR 8211 - EHESS, Université Paris Descartes ; brigitte.chamak@parisdescartes.fr 
sur Internet des associations de personnes autistes et des blogs qui rendent compte de leur expérience et de leurs revendications permet de mesurer l'ampleur du phénomène qui se situe dans le contexte du développement des disability studies, recherches qui donnent une place centrale aux personnes concernées. Ce courant prend de l'ampleur au niveau international et participe aux changements des représentations. Entre adaptation, négociation et résistance, les initiatives portées par les associations ou les collectifs, produisent des pratiques concrètes qui bouleversent les représentations et les politiques publiques. Nick Crossley (2006), pionnier de l'analyse culturelle des mouvements sociaux, a étudié le champ de la contestation psychiatrique au Royaume-Uni et a montré comment les revendications de groupes sociaux spécifiques s'incarnaient sous la forme d'une action politique. Dans le cas de l'autisme, des collectifs se sont constitués autour d'affiliations identitaires et culturelles, la recherche des causes structurelles de la stigmatisation dans la société, et la volonté de trouver des solutions adaptées à leurs difficultés. Mon travail a consisté à analyser ces transformations en France tout en comparant les représentations de l'autisme dans d'autres cultures et dans un contexte international.

\section{Les changements de définition et la diversité des représentations culturelles}

Au cours des vingt dernières années, la construction sociale de l'autisme a subi de grandes transformations au niveau international (Chamak, 2008 ; Eyal et coll. 2010 ; Grinker, 2007 ; Hacking, 2009 ; Murray, 2008 ; Nadesan, 2005). Sa définition, les hypothèses étiologiques et les traitements ont changé. L'autisme est passé du statut de maladie rare à celui de syndrome fréquent incluant des formes très diverses et de la catégorie de trouble psychiatrique à celui de maladie d'origine génétique impliquant un développement atypique du système nerveux (Bumiller, 2009 ; Hacking, 2009 ; Murray, 2008 ; Nadesan, 2005). Les parents ont adopté ces explications biologiques qui contribuaient à diminuer leur sentiment de culpabilité (Chamak, 2008 ; Farrugia, 2009). Dans les années 1960, un discours psychanalytique avait construit l'autisme comme le résultat d'un comportement inadéquat des parents, et plus particulièrement de la mère. Cette théorie psychogénétique a été combattue dès 1964 par Bernard Rimland, psychologue, lui-même père d'un enfant autiste, qui a proposé une explication alternative basée sur la neurobiologie et qui a fondé en 1965 l'American Society for Autism. Depuis les années 1990, la majorité des associations de parents d'enfants autistes adoptent la vision et les discours des neuroscientifiques et des généticiens pour redéfinir l'autisme comme un problème neurodéveloppemental présentant des composantes génétiques fortes (Caruso, 2010 ; Chamak, 2008).

Les imaginaires nourris de contes de fées, incluant la substitution d'un bébé par celui d'une fée, et les clichés, préjugés, stéréotypes, croyances, comme celle d'un enfant ensorcelé par les esprits (Brauner et Brauner, 1986 ; Grinker, 2007), ou les métaphores inspirées de la science-fiction, ont façonné les représentations populaires de l'autiste entre "idiot», "possédé », " élu », " alien » ou « génie » (Caruso, 2010 ; Hacking, 2009 ; Murray, 2008). Stuart Murray (2008), pour qui parler d'autisme, c'est traiter de la condition humaine, a exploré les mécanismes culturels par lesquels l'autisme est devenu un sujet de fascination. En Afrique, les autistes sont parfois perçus comme des idiots, victimes des fautes commises par ses parents ou par d'autres membres de sa famille (Ebwel et coll. 2010). Certains sont considérés comme des sorciers, comme un porte-bonheur, ou, au contraire, comme une malédiction. L'enfant est supposé occuper une place importante dans le monde invisible où il aurait un autre statut que celui qu'il a dans le monde visible des vivants. Seules les personnes qui détiennent une puissance surnaturelle (sorciers, prêtres ou pasteurs) seraient à même de traiter cet enfant possédé par des forces maléfiques. Souvent, les parents sont jugés coupables du trouble de leur enfant, ou encore comme les cibles ayant occasionné le mécontentement des ancêtres. La thérapie consiste à soumettre les parents et l'enfant aux séances d'exorcisme à travers les prières, en parallèle de l'approche biomédicale qui n'est pas exclue, surtout en milieu urbain. Chez les Pygmées d'Afrique centrale, Grinker (2007) a remarqué que quand un enfant ne parlait pas, ne regardait pas dans les yeux et commençait à avoir des épilepsies, ses parents supposaient qu'il était attaqué par des ancêtres. Ils lui 
donnaient des herbes médicinales et l'envoyaient loin du village, là où il n'aurait de contact avec aucun membre de la famille.

Dans leur étude sur les modèles explicatifs de dix parents d'enfants autistes originaires du Maghreb vivant au Québec, Imen Ben-Cheikh et Cécile Rousseau (2013) ont montré que les causes biomédicales prédominaient et étaient citées en premier mais tous les parents nommaient au moins deux systèmes explicatifs appartenant au registre des croyances biomédicales et religieuses. Des croyances traditionnelles étaient également évoquées ( «mauvais œil », sorcellerie). Ces modèles explicatifs coexistaient dans un même récit avec davantage de questionnement que de certitudes. Bien que toutes les mères contactées aient accepté les thérapies comportementales utilisées au Canada, quelques-unes ont exprimé un certain malaise par rapport à ces méthodes perçues comme trop exigeantes pour leur enfant. Dans son article sur la prise en compte de la dimension culturelle dans l'accompagnement d'un enfant autiste d'origine kabyle dans un jardin d'enfants thérapeutique en France, Valérie Rousselon (2012) montre que donner l'opportunité aux parents d'origine étrangère de s'exprimer sur leurs conceptions les soulage et permet de mieux comprendre la complexité des histoires cliniques.

En Corée, les enfants autistes sont souvent cachés, en grande partie à cause de la stigmatisation associée au fait d'avoir un enfant présentant un handicap (Grinker, 2007). Une étude réalisée sur 55000 enfants de 7 à 12 ans dans une communauté en Corée du sud indique que deux tiers des cas d'autisme se retrouvent à l'école normale (Kim et coll., 2011). Ces cas ne sont pas diagnostiqués compte tenu de la réticence des parents à parler des problèmes de leur enfant. De la même façon, en Iran, le faible taux de prévalence de l'autisme serait lié à l'importante stigmatisation associée au fait d'avoir un enfant avec un handicap (Samadi et coll., 2012). Les parents ne décrivent pas les difficultés rencontrées par leurs enfants pour éviter les écoles spécialisées.

Dans la communauté ultraorthodoxe en Israël, la perception de l'autisme fait appel à des références très diverses qui vont de la biomédecine aux cadres religieux et spirituels, ainsi qu'aux traitements alternatifs (homéopathie, réflexologie, biofeedback, chiropractie, delphinothérapie...) (Shaked, Bilu, 2006). Déçues par les succès limités des différentes approches, les mères cherchent des explications métaphysiques dans leur système de référence religieux et font souvent appel à la communication facilitée, interprétant les écrits comme la faculté de l'enfant à communiquer avec l'au-delà. Les explications naturalistes et métaphysiques coexistent. La dualité corps-esprit se concrétise par des explications qui ont trait à la fois au cerveau endommagé mais aussi à l'âme spécialement élevée chez ces enfants. Les mères ultraorthodoxes oscillent entre images négatives de leur enfant, diffusées par les conceptions naturalistes de l'autisme, et images positives soutenues par des sources mystiques.

Les représentations de l'autisme, et plus généralement des personnes présentant un handicap, véhiculées par les médias renforcent souvent les stéréotypes. Ainsi, l'étude de Jones et Harwood (2009) sur les représentations de l'autisme dans la presse écrite australienne entre 1996 et 2005 montre que l'autiste y est présenté en général de façon négative, soit comme un objet de pitié, comme un poids pour sa famille, le système éducatif et la société dans son ensemble, voire comme un être agressif, dangereux et incontrôlable ou comme une personne étrange mais présentant certaines capacités exceptionnelles, à l'image de Rain man.

\section{La neurodiversité : l'introduction de nouveaux acteurs}

En intégrant des personnes capables de s'exprimer, la nouvelle définition de l'autisme a permis la prolifération des témoignages, d'abord celui de Temple Grandin (1986), puis celui de Donna Williams (1992) et de nombreuses autres autobiographies ont été publiées par la suite (Chamak, 2005). Les échanges sur Internet entre personnes autistes se sont également multipliés et ont favorisé l'émergence d'associations, dont la première ANI (Autism Network International) a été créée en 1991 (Sinclair, 2005). L'impact social des transformations des représentations de l'autisme induites par les changements des classifications, la diffusion des témoignages et la création de nouveaux sites sur Internet ont participé au développement 
d'une nouvelle culture de l'autisme et du passage d'une vision négative et stigmatisante à une représentation positive et valorisante du fonctionnement autistique.

Le concept de neurodiversité, conçu dans les années 1990 par l'australienne Judy Singer, a été rapidement diffusé par les médias et a alimenté la réflexion des réseaux associatifs qui se sont constitués dans une logique d'empowerment pour développer une politique identitaire et produire un discours de type culturaliste insistant sur les aspects positifs de la différence. Les disability studies, qui développent un modèle social du handicap remettant en question la représentation du handicap comme un problème individuel d'ordre médical, ont nourri les nouvelles représentations diffusées sur les sites et les blogs des associations de personnes autistes.

Katherine Seidel, fondatrice du site Neurodiversity.com, cherche ainsi à généraliser l'expression « différence cognitive » pour tous ceux actuellement stigmatisés par les étiquettes " déviance », «trouble » ou « syndrome » (Seidel, 2004). Une nouvelle rhétorique s'est forgée et s'est généralisée parmi les personnes diagnostiquées avec un " problème de santé mentale ». Plutôt que d'être stigmatisées comme ayant un problème psychiatrique, elles choisissent le terme "neurodiversité » et se présentent comme ayant un cerveau qui fonctionne différemment (Antonetta, 2005). En 2009, s'est créée à Londres l'association DANDA (Developmental Adult Neuro-Diversity Association) qui cherche à regrouper des adultes présentant diverses problématiques (dyspraxie, dyslexie, dyscalculie, syndrome d'Asperger, hyperactivité, etc.) afin de les aider à s'impliquer dans des activités d'interactions et d'entraide. Depuis 2004, Michelle Dawson s'est érigée contre la présentation de l'autisme comme une catastrophe et a développé un argumentaire très détaillé remettant en question les méthodes comportementales. Plus récemment, Nick Walker (2013) insiste sur la nécessité d'abandonner la notion de pathologie. Sur son blog Neurocosmopolitanism, il cite les travaux de Sarah Coenen and Helen Cha-Choe, qui utilisent le paradigme de la neurodiversité pour explorer les attitudes des cliniciens qui travaillent avec des personnes autistes. Pour elles, il est évident que traiter des personnes autistes comme "déficientes " relève de préjugés et que le paradigme de la pathologie crée des attitudes condescendantes et dédaigneuses qui sont vécues comme des agressions.

La parole et les revendications des personnes autistes concernant leur propre expertise dans la production des connaissances sur l'autisme ne sont que très récemment prises en compte. Damian Milton (2014) signale la méfiance des personnes autistes vis-à-vis des chercheurs et de leurs motivations compte tenu du décalage entre leurs besoins et les priorités de la recherche scientifique. II estime que la principale mission pour ceux qui tentent de travailler avec des personnes autistes est d'apprécier les connaissances spécifiques qu'elles possèdent afin d'interagir de façon plus constructive.

Les interlocuteurs autistes se sont multipliés ces dernières années comme en attestent les textes sur internet (Dawson, 2004 ; Sinclair, 2005), les différentes associations (ANI, Autistic Self Advocacy Network, Aspies For Freedom, Autism Rights Movement, SAtedl), les videoblogs sur YouTube (Ben Belek, 2013), les articles et les livres rédigés par des personnes autistes (Miller, 2003 ; Milton, 2014). Aux USA, Ari Ne'eman fondateur d'ASAN (Autistic Self Advocacy Network), a été nommé en 2010 au conseil national sur le handicap. En France, les membres de l'association SAtedl (Spectre Autistique troubles envahissants du développement International) sont consultés par les pouvoirs publics.

\section{La production d'environnements favorables et les débouchés professionnels}

Le concept «autism-friendly » qui implique des modifications de l'espace physique et des modes d'interactions plus adaptés pour prendre en compte le fonctionnement et les sensibilités particulières des autistes, commence à se diffuser (Nguyen, 2006). Aux États-Unis, le Theatre Development Fund a créé un programme en 2011 pour rendre le théâtre accessible aux enfants et aux adultes autistes. Cette initiative fait partie du programme d'accessibilité (en lien avec l'association Autism Speaks et Dysney). À Londres, les cinémas Odeon ont mis en œuvre des nuits "sensory friendly". L'expansion des marchés associés à l'autisme est impressionnante: avec du temps et de l'argent, des services et des produits "autism- 
friendly » de toutes sortes peuvent être trouvés, des leçons de ski aux croisières en passant par des régimes alimentaires spéciaux (Margolies, 2008).

Les nouvelles représentations de l'autisme sont à l'origine d'initiatives d'embauche mais toujours pour des personnes qui peuvent prouver leurs capacités à répondre aux besoins de l'entreprise. En mai 2013, l'entreprise SAP, leader sur le marché des logiciels d'entreprise, a lancé l'initiative « Autism at work » et s'est engagée à recruter plus de 650 personnes autistes d'ici 2020. SAP a passé un accord avec Specialisterne, cabinet de recrutement suédois fondé par le père d'un enfant autiste et spécialisé dans la valorisation des compétences et le recrutement des personnes autistes. Il est toutefois important de souligner que la sélection est rude et que seules les personnes sans déficience intellectuelle et avec des capacités particulières peuvent accéder à ce type d'emplois.

En France, l'insertion professionnelle des personnes handicapées passe par des structures telles que les entreprises adaptées et les établissements et services d'aide par le travail. C'est à ce titre qu'en 2013, l'entreprise adaptée Socia3, filiale du groupe Soregor, spécialisé dans l'externalisation des fonctions administratives, a lancé son projet intitulé « Hors les Murs » qui vise l'insertion professionnelle de personnes autistes.

Des initiatives très récentes émergent dans le cadre associatif créé par des personnes autistes en France. L'association Talent d'AS ${ }^{1}$ se consacre « à faire progresser le travail et l'autonomie des adultes autistes et asperger, notamment dans l'emploi en milieu ordinaire ». L'association $A S P^{\prime} I D^{2}$ a également pour objectif « l'épanouissement personnel, social et professionnel 》 des personnes autistes sans déficience intellectuelle. Leur projet s'articule autour de différentes actions : la mise en place d'une pépinière de talents, de « mentorats pair-à-pair » basés sur la "pair-émulation » et l'élaboration d'un plan de communication favorisant les rencontres entre personnes autistes et entreprises. Partant du principe que « les personnes avec autisme « ont des passions, des centres d'intérêts restreints et des talents bien souvent mal-exploités ", il s'agit pour ASP'ID de mettre en place un lieu de rassemblement, où les personnes apprennent à développer leur passion, à la partager avec les autres, et à découvrir leur potentiel professionnel. Cependant, le constat est toujours le même : ceux qui présentent les plus grandes difficultés ne trouvent pas de solution et ce sont les familles qui doivent assumer, souvent seules, les problèmes soulevés par un adulte dépendant (Howlin et coll., 2013).

\section{Conclusion}

En 2010, Daniela Caruso, professeur de droit à l'université de Boston, constatait que l'autisme a transformé la façon dont nous pensons le handicap. Quand l'autisme est perçu comme une catastrophe, les associations de parents insistent sur l'urgence de la recherche, les traitements et leurs coûts, mais quand l'accent est mis sur la beauté et le génie, l'autisme est vu comme un investissement. Des personnes autistes prennent la parole, s'auto-organisent, tiennent un discours politique et contestataire, revendiquent le droit à la citoyenneté et s'exposent sur des vidéoblogs pour faire part de leur expérience (Ben Belek, 2013).

Les changements de représentations de l'autisme, et plus largement du handicap, n'impliquent pas la disparition des anciennes représentations qui coexistent à des degrés divers et sont différentes en fonction des pays, des cultures et des familles. Les nouvelles représentations qui visent à déstigmatiser l'autisme permettent à certaines personnes de réinterprétrer leur vie avec une nouvelle grille de lecture, surtout depuis que le paradigme de la neurodiversité s'est propagé. Le diagnostic de syndrome d'Asperger illustre les effets de looping décrits par le philosophe lan Hacking (1995) puisqu'il peut changer la compréhension et les représentations des personnes diagnostiquées. Mais si ceux qui ont volontairement adopté ce diagnostic se sentent mieux, la stigmatisation est bien plus importante pour les enfants et les adolescents qui, autrefois, étaient qualifiés d'introvertis étranges mais doués et qui, aujourd'hui, sont considérés comme souffrant de troubles autistiques.

\footnotetext{
${ }^{1} \mathrm{https}: / /$ talentdas.wordpress.com/

2 http://www.asp-id.com/
} 
Les chercheurs en sciences cognitives se focalisent davantage sur le syndrome d'Asperger et de moins en moins de professionnels s'intéressent à ceux qui ne présentent pas leurs compétences. Les écoles et certains établissements spécialisés préfèrent accueillir des enfants qui parlent et qui sont intelligents plutôt que des enfants sans langage qui ont de graves troubles de comportement. Ainsi, ceux qui constituaient le cœur de l'autisme, autrefois, s'en trouvent peu à peu écartés parce que moins faciles à gérer et plus difficiles à faire progresser. Pour les parents vieillissants qui s'occupent de leur enfant adulte dépendant, les inquiétudes sur le devenir de leur enfant s'accroissent car la déstigmatisation ne concerne pas les personnes présentant le plus de difficultés. Au contraire, une compétition pour sélectionner les plus performants est à l'œuvre comme le montre la sélection à l'embauche ou à l'admission, même dans des structures spécialisées comme les établissements et services d'aide par le travail.

En analysant les nouvelles figures du handicap, Anne Marcellini (2007) a montré que si l'hypothèse d'un processus global de déstigmatisation à l'œuvre au cours du XXe siècle pouvait être soutenue, la figure de la "réparation technologique de l'humain », figure valorisée et dont le sportif handicapé est emblématique, dessine les contours et les limites de ce processus. La rencontre entre handicap et activités sportives se développe de plus en plus dans une logique sportive valorisant la compétition en référence au monde de l'excellence et de la performance dans un processus d'aspiration vers la norme sportive et dans une logique d'efficacité et de concurrence. Les figures sportives les plus médiatisées sont celles des sportifs appareillés de flexfoot (lames courtes en graphite carbone utilisées comme prothèses), mises en scène du corps handicapé sportif, actif, technologique, incarnant la figure du dépassement de soi mais aussi de la valorisation des technosciences (Marcellini, 2007).

De nombreux auteurs se sont intéressés aux représentations sociales du handicap depuis les années 1980 (Gardou, 2010 ; Sticker, 2009) insistant sur la différenciation radicale entre atteinte " mentale » et « physique » et soulignant le discrédit associé à l'attribut " handicap » qui mérite alors l'appellation de stigmate (Goffman, 1975) avec une surstigmatisation du handicap mental. Mais les dernières décennies ont vu s'organiser la prise de parole des personnes stigmatisées, en rupture le plus souvent avec les discours d'experts, remettant en question les catégories, les conceptions, les approches et le vocabulaire de ceux qui se disent spécialistes (Chamak, 2010 ; Milton, 2014). Le discours biologique et médical prédominant est contesté. La vision sociale du handicap se répand, notamment par la diffusion des disability studies (Barnes, 2012). Comme le mouvement des sourds, celui des personnes autistes remet en cause les définitions médicales du handicap et l'idée même de déficience ou de handicap. Une identité collective se construit autour d'expériences et d'intérêts communs. La perception d'un statut partagé, de difficultés communes, le sentiment d'être discriminé, participent à cette construction et à la constitution de cette communauté qui se modifie en échangeant et qui tente de transformer certaines représentations et normes de la société.

\section{Bibliographie}

ANTONETTA, S. 2005. A mind apart: travels in a neurodiverse world, New York, Penguin.

BARNES, C. 2012. "The social model of disability : valuable or irrelevant ? ", dans N. Watson, A. Roulston, C. Thomas (sous la direction de), The Routledge Handbook of Disability Studies, Londres, Routledge, p. 12-29.

BELEK, B. 2013. "I Believe it can change the way things are ". Identity construction among video-bloggers with Asperger's syndrome on YouTube, Diemen (Pays-Bas), AMB.

BEN-CHEIKH, I. ; ROUSSEAU, C. 2013. "Autisme chez des enfants d'immigration récente: modèles explicatifs de familles originaires du Maghreb ", L'Autre, 14, p. 213-222.

BRAUNER, A. ; BRAUnER, F. 1986. L'enfant déréel : histoire des autismes depuis les contes de fées, Toulouse, Privat.

BUMILLER, K. 2009. " The Geneticization of Autism: From New Reproductive Technologies to the Conception of Genetic Normalcy ", Signs : Journal of Women in Culture and Society, 34, p. 875-899.

CARUSO, D. 2010. "Autism in the US: social movement and legal change », American Journal of Law and Legal Change, 36, p. 483-539. 
CHAMAK, B. 2005. "Les récits de personnes autistes : une analyse socio-anthropologique ", Handicap - revue de sciences humaines et sociales, 105-106, p. 33-50.

CHAMAK, B. 2008. "Autism and Social Movements: French Parents'Associations and International Autistic Individuals'Organizations ", Sociology of Health and IIIness, 30, p. 76-96. CHAMAK, B. 2010. "Autisme, handicap et mouvements sociaux », Alter, European Journal of Disability Research, 4, p. 103-115.

CHAMAK, B. 2013. "L'autisme à l'écran ». Consulté le 01/03/2018. https://halshs.archivesouvertes.fr/halshs-01182764/document

CROSSLEY, N. 2006. "Changement culturel et mobilisation des patients. Le champ de la contestation psychiatrique au Royaume-Uni, 1970-2000 », Politix, 73, p. 33-55.

DAWSON, M. 2004. "The misbehaviours of behaviorists: ethical challenges to the autism-ABA industry ». Consulté le 01/03/2018. http://www.sentex.net/ nexus23/naa aba.html

EBWEL, J.M. ; ROEYERS, H. ; DeVLIEGER, P. 2010. " Approches des représentations sociales de l'autisme en Afrique ", enfances\&PSY, $n^{\circ} 49$, p. 121-129.

EYAL, G. ; HART, B. et coll. 2010. The Autism Matrix: The Social Origins of the Autism Epidemic, Cambridge, Polity.

FARRUGIA, D. 2009. « Exploring Stigma: Medical Knowledge and the Stigmatisation of Parents of Children Diagnosed with Autism Spectrum Disorder », Sociology of Health and IIIness, 31, p. 1011-1027.

GARDOU, C. (sous la direction de) 2010. Le handicap au risque des cultures. Variations anthropologiques, Toulouse, érès.

GofFMAN, E. 1975. Stigmate. Les usages sociaux des handicaps, Paris, Les Éditions de Minuit.

GRANDIN, T. 1986. Ma vie d'autiste, Paris, Odile Jacob.

GRINKER, R.R. 2007. Unstrange Minds: Remapping the World of Autism, Cambridge, MA, Basic Books.

HACKING, I. 1995. "The looping effects of human kinds ", dans D. Sperber, D. Premack, A. Premack (sous la direction de), Causal Cognition: A Multidisciplinary Debate, New York, Oxford University Press, p. 351-383.

HACKING, I. 2009. « Humans, aliens \& autism », Daedalus, 138, p. 44-59.

HowLIN, P. ; Moss, P. et coll. 2013. "Social outcomes in mid to later adulthood among individuals diagnosed with autism and average nonverbal IQ as children ", Journal of American Academy of Child and Adolescent Psychiatry, 52 (6), p. 572-581.

JONES, S. ; HARWOOD, V. 2009. "Representations of autism in Australian print media ", Disability \& Society, 24, p. 5-18.

KIM, Y.S. ; BENNETT, L. et coll. 2011. «Prevalence of Autism Spectrum Disorders in a Total Population Sample », American Journal of Psychiatry, 168, p. 904-912.

MARCELLINI, A. 2007. "Nouvelles figures du handicap? Catégorisations sociales et dynamiques des processus de stigmatisation/déstigmatisation », dans G. Boëtsch, C. Hervé, J. Rozenberg (sous la direction de), Corps normalisé, corps stigmatisé, corps racialisé, Louvain-La-Neuve: De Boeck, p. 199-219.

MARGOLIES, J. 2008. «Bypassing the roadblock of autism ", New York Times. http://www.nytimes.com/2008/09/14/travel/14heads.html? $\quad \mathrm{r}=0$ \&pagewanted=print. Consulté le 01/03/2018.

Miller, J.K. 2003. Women From Another Planet? Our Lives in the Universe of Autism, Bloomington, $1^{\text {st }}$ Books Library.

MILTON, D. 2014. "Autistic expertise: a critical reflection on the production of knowledge in autism studies », Autism, 18, p. 794-802.

MURRAY, S. 2008. Representing autism: culture, narrative, fascination, Liverpool, Liverpool University Press.

NADESAN, M. 2005. Constructing Autism. Unravelling the 'Truth' and Understanding the Social. Londres, New York, Routledge.

NGUYEN, A. 2006. Creating an Autism Friendly Environment, U.K., National Autistic Society. ORTEGA, M. 2009. "The cerebral subject and the challenge of neurodiversity ", BioSocieties, $4,425-445$. 
ROUSSELON, V. 2012. "Les dimensions culturelles de la culpabilité : prise en charge institutionnelle d'un enfant autiste et de sa famille d'origine kabyle ", dans S. Missonnier (sous la direction de), Honte et culpabilité dans la clinique du handicap, p. 123-138.

SAMAdI, S.A.; MAHMOODIZADEH, A.; MCCONKEY, R. 2012. "A National Study of the Prevalence of Autism among Five-Year-Old Children in Iran », Autism, 16, 5-14.

SEIDEL, K. 2004. "The autistic distinction». Consulté le 13 janvier 2015. http://www.neurodiversity.com/autistic distinction.html.

SHAKED, M. ; BILU, Y. 2006. "Grappling with Affliction: Autism in the Jewish Ultraorthodox community in Israel », Culture, Medicine, and Psychiatry, 30, p. 1-27.

SINCLAIR, J. 2005. "Autism Network International: the development of a community and its culture ». Consulté le 25 mars 2015. http://www.autreat.com/History of ANI.html.

STICKER, H.-J. 2009. Les métamorphoses du handicap de 1970 à nos jours, Grenoble, Presses Universitaires de Grenoble.

WALKER, N. 2013. "Throw away the master's tools: liberating ourselves from the pathology paradigm ». Consulté le 25 mars 2015. http://neurocosmopolitanism.com/throw-away-themasters-tools-liberating-ourselves-from-the-pathology-paradigm/.

WILLIAMS, D. 1992. Nobody Nowhere: The Extraordinary Autobiography of an Autistic, New York, Time Books.

\section{Résumé}

Depuis les années 1990, les représentations sociales de l'autisme ont subi de profondes transformations, même si la diversité des représentations culturelles demeure. La définition de l'autisme, les hypothèses étiologiques et les traitements ont changé. L'élargissement des critères diagnostiques est à l'origine de l'introduction dans la catégorie de personnes avec langage aux capacités cognitives importantes. Ces personnes ont commencé à s'exprimer et redéfinir l'autisme comme une différence et non comme une maladie ou un handicap. Un nouveau mouvement social célèbre la "neurodiversité », participe à une de-stigmatisation de l'autisme et à l'apparition du concept "autism-friendly ». Cependant, ces représentations positives et la médiatisation accrue des personnes présentant un syndrome d'Asperger ont pour conséquence un désintérêt pour ceux qui n'ont pas leurs compétences.

Mots-clés : autisme, représentations, neurodiversité, autism-friendly.

\section{Summary}

Changes in social representations of autism and the autism-friendly concept

Since the 1990s, social representations of autism have undergone profound changes, although there is still a wide diversity according to cultures. The definition of autism, the aetiological hypotheses and the treatments have all changed. The broadening of diagnostic criteria has led to the inclusion of people with speaking skills and cognitive abilities in this category. These different subjects have begun to speak out, and to give another perspective on autism, treating it as a difference rather than a disease or a disability. A new social movement has emerged, laying claim to "neurodiversity" and autism-friendly environment, contributing to the destigmatisation of autism. However, these positive representations and the increased media coverage devoted to people with Asperger syndrome have resulted in a loss in interest for those who do not possess the same abilities.

Key words : Autism, representations, neurodiversity, autism-friendly. 